\title{
The first Conus genome assembly reveals a primary genetic central dogma of conopeptides in C. betulinus
}

Chao Peng ${ }^{1,2}$, Yu Huang $\mathbb{1}^{1,3}$, Chao Bian ${ }^{1,3,4}$, Jia Li ${ }^{1}$, Jie Liu ${ }^{5,6}$, Kai Zhang ${ }^{1,3,10}$, Xinxin You ${ }^{1,3}$, Zhilong Lin ${ }^{5}$, Yanbin He ${ }^{5}$, Jieming Chen ${ }^{1,3}$, Yunyun Lv ${ }^{1,3,1}$, Zhiqiang Ruan ${ }^{1,3}$, Xinhui Zhang ${ }^{1}$, Yunhai Yi $\mathbb{B}^{1,3}$, Yanping Li, ${ }^{1,11}$, Xueqiang Lin ${ }^{1}$, Ruobo Gu${ }^{1}$, Junmin Xu', Jia'an Yang ${ }^{7}$, Chongxu Fan ${ }^{8}, \mathrm{Ge} \mathrm{Yao}^{8}$, Ji-Sheng Chen ${ }^{8}$, Hui Jiang ${ }^{8}$, Bingmiao Gao ${ }^{2}$ and Qiong Shi $\mathbb{1}^{1,3,9}$

\begin{abstract}
Although there are various Conus species with publicly available transcriptome and proteome data, no genome assembly has been reported yet. Here, using Chinese tubular cone snail (C. betulinus) as a representative, we sequenced and assembled the first Conus genome with original identification of 133 genome-widely distributed conopeptide genes. After integration of our genomics, transcriptomics, and peptidomics data in the same species, we established a primary genetic central dogma of diverse conopeptides, assuming a rough number ratio of 1:1:1:10s for the total genes: transcripts: proteins: post-translationally modified peptides. This ratio may be special for this wormhunting Conus species, due to the high diversity of various Conus genomes and the big number ranges of conopeptide genes, transcripts, and peptides in previous reports of diverse Conus species. Only a fraction (45.9\%) of the identified conotopeptide genes from our achieved genome assembly are transcribed with transcriptomic evidence, and few genes individually correspond to multiple transcripts possibly due to intraspecies or mutationbased variances. Variable peptide processing at the proteomic level, generating a big diversity of venom conopeptides with alternative cleavage sites, post-translational modifications, and $\mathrm{N}$-/C-terminal truncations, may explain how the 133 genes and $\sim 123$ transcripts can generate thousands of conopeptides in the venom of individual C. betulinus. We also predicted many conopeptides with high stereostructural similarities to the putative analgesic $\omega$-MVIIA, addiction therapy AulB and insecticide Iml, suggesting that our current genome assembly for C. betulinus is a valuable genetic resource for high-throughput prediction and development of potential pharmaceuticals.
\end{abstract}

Correspondence: Bingmiao Gao (gaobingmiao@hainmc.edu.cn) or Qiong Shi (shiqiong@genomics.cn)

'Shenzhen Key Lab of Marine Genomics, Guangdong Provincial Key Lab of Molecular Breeding in Marine Economic Animals, BGl Academy of Marine Sciences, BGI Marine, BGI, Shenzhen, Guangdong 518083, China

${ }^{2}$ Key Laboratory of Tropical Translational Medicine of Ministry of Education, Hainan Provincial Key Laboratory of Research and Development of Herbs, School of Pharmacy, Hainan Medical University, Haikou, Hainan 570102, China Full list of author information is available at the end of the article These authors contributed equally: Chao Peng, Yu Huang, Chao Bian, Jia Li, Jie Liu, Kai Zhang

\section{Introduction}

Cone snails (Conus spp.) are a large genus of gastropods that feed on a variety of prey, including worms, snails, and fishes $^{1,2}$. Given that there are 700 Conus species around the world and each possesses over 100 various conopeptides (collectively known as small, bioactive, and heavily post-translationally modified peptides in the Conus venom $)^{1-4}$, it has been estimated that there are over 80,000 natural conopeptides ${ }^{1,5}$, of which some have been approved as valuable pharmacological probes and clinical drugs such as the well-known $\omega$-MVIIA (Ziconotide) for

\section{(c) The Author(s) 2021}

(c) (i) Open Access This article is licensed under a Creative Commons Attribution 4.0 International License, which permits use, sharing, adaptation, distribution and reproduction cc) in any medium or format, as long as you give appropriate credit to the original author(s) and the source, provide a link to the Creative Commons license, and indicate if changes were made. The images or other third party material in this article are included in the article's Creative Commons license, unless indicated otherwise in a credit line to the material. If material is not included in the article's Creative Commons license and your intended use is not permitted by statutory regulation or exceeds the permitted use, you will need to obtain permission directly from the copyright holder. To view a copy of this license, visit http://creativecommons.org/licenses/by/4.0/. 
treatment of chronic pain in cancer patients ${ }^{6}$. Usually, conopepetides are synthesized in the venom gland as precursor proteins from a single gene that is often comprised of a highly conserved signal peptide, a pro-peptide region, and a hypervariable mature peptide sequence ${ }^{7}$; they have been classified into various superfamilies based on the sequence similarities of their signal peptides.

To date, hundreds of conopeptide genes or conopeptide coding sequences (CDS) have been identified by PCR amplification or purified from crude venom of cone snails using mass chromatography (MS). Moreover, recent highthroughput pipelines of integrating multi-omics (such as transcriptomics and peptidomics ${ }^{4,8,9}$ ) have dramatically facilitated the discovery of novel conopeptides. In fact, it has been estimated that much less $(<2 \%)$ of the total conopeptide diversity has been sequenced ${ }^{10}$. Recently, employing advanced MS systems, several research teams ${ }^{9,11,12}$ have reported the presence of over 1000 different peptides in a single venom, which is a remarkable increase from the early popular estimates of 50-200 conopetides per species. However, great difficulty in extraction of high-quality genomic DNAs has hindered whole-genome sequencing of cone snails ${ }^{1}$, which should have become a valuable resource for comparative examinations of detailed transcription and translation of conopetide genes in single or different Conus species.

In 2011, a trial next-generation shotgun survey with low quality for C. bullataus genome was reported ${ }^{13}$. Recently, targeted sequencing of venom genes from 32 Conus genomes $^{14}$ characterized various conopeptide superfamilies. Unpublished genome data for C. consors were also deposited at NCBI (under the accession number GCA_004193615.1) for public availability. Here, we generated the first genome assembly for the predominant vermivorous Chinese tubular cone snail (C. betulinus; Fig. 1a) in the South China Sea to shed light on the representative genome structure of Conus species, and attempted to illustrate a preliminary genetic central dogma of conopeptides for high-throughput development of novel marine drugs. By integration of present genomics and peptidomics data, along with our previously published transcriptomics results in the same species, we tried to establish a rough number ratio for the total genes/transcripts/proteins/post-translationally modified peptides in this representative Conus species. Our achieved genome assembly will definitely become a valuable genetic resource for high-throughput prediction of conopeptide genes and transcripts, and lay a solid foundation for in-depth investigations on Conus biology and conopeptide pharmacology.

\section{Results}

\section{Generation of the first Conus genome assembly}

Specimens of wild middle body-sized ${ }^{4}(8.5 \pm 0.5 \mathrm{~cm}$ in length) $C$. betulinus were collected in the offshore areas of
Sanya City, Hainan Province, China. Seven Illumina libraries with various insert-sizes (short: 250, 500, and $800 \mathrm{bp}$; long: 2, 5, 10, and $20 \mathrm{~kb}$ ) were constructed ${ }^{15}$ using extracted genomic DNAs from a pool of muscle samples, and then they were sequenced on an Illumina HiSeq 2000 platform (San Diego, CA, USA). After removal of adapter sequences, low-quality raw reads, and PCR duplicates ${ }^{15}$, a total of $188.05-\mathrm{Gb}$ clean reads $(59.65 \%$ of the $315.24-\mathrm{Gb}$ raw reads) were retained (Supplementary Table S1) for subsequent assembly. Meanwhile, after correction with Illumina short-insert reads, we obtained $225.39 \mathrm{~Gb}$ of PacBio corrected long reads $(94.26 \%$ of the $239.69-\mathrm{Gb}$ raw reads; Supplementary Table S2).

A routine hybrid strategy ${ }^{16}$ was employed to assemble the genome of $C$. betulinus. The final assembly of $3.43 \mathrm{~Gb}$ in size, similar to the reported Conus estimates by fluorometric assays ${ }^{14}$, accounted for $86.00 \%$ of the estimated genome size $(3.99 \mathrm{~Gb})$ by a genome survey ${ }^{15}$ in the present study (Fig. 1b; Supplementary Table S3). Its scaffold N50 and contig N50 values are $232.61 \mathrm{~kb}$ and $171.48 \mathrm{~kb}$, respectively (Table 1); the genome GC content is $43.80 \%$ (Supplementary Fig. S1), and transposable elements (TEs) comprise $38.56 \%(1.32 \mathrm{~Gb})$ of the achieved genome assembly (Supplementary Table S4). We also evaluated the completeness of our C. betulinus assembly with metazoan benchmarking universal single-copy orthologs (BUSCOs) ${ }^{17}$. The high BUSCO value (Supplementary Fig. S2) suggests that our assembled genome covers at least $89.8 \%$ of the gene space ${ }^{15}$. Based on the routine annotation strategy ${ }^{15}$ using a combination of transcriptome-based, homologous, and ab initio predictions, we annotated 22,698 protein-coding genes in the genome assembly (Supplementary Table S5).

\section{Original identification of 133 genome-widely distributed conopeptide genes}

After sequence alignment of our previously identified 215 conopeptide transcripts (from a pool of various individuals and tissues; called as Bt001-Bt215) ${ }^{4}$ against the achieved genome assembly, we eventually predicted a total of 133 conopeptide genes (named as conot001conot133) with complete sequences of 1-4 exons (Fig. 1c-e; Supplementary Table S6, Fig. S3). The exon sizes range from 14 to $345 \mathrm{bp}$, with an average length of $87 \mathrm{bp}$ (Fig. 1f).

These predicted conopeptide genes were deduced to corresponding protein sequences, and therefore they are named as "CONOT001-CONOT133" (Supplementary Table S7). Proteins of the same genes and transcripts are termed in the format of "CONOTx(Bty)" ( $\mathrm{x}$ and $\mathrm{y}$ refer to Arabic number suffixes) such as CONOT103(Bt176). These deduced conopeptide proteins can be classified ${ }^{4,9}$ into 21 known superfamilies, 1 cysteine-rich family (Conkunitzin), 1 non-cysteine family (Conantokin-like), 
a

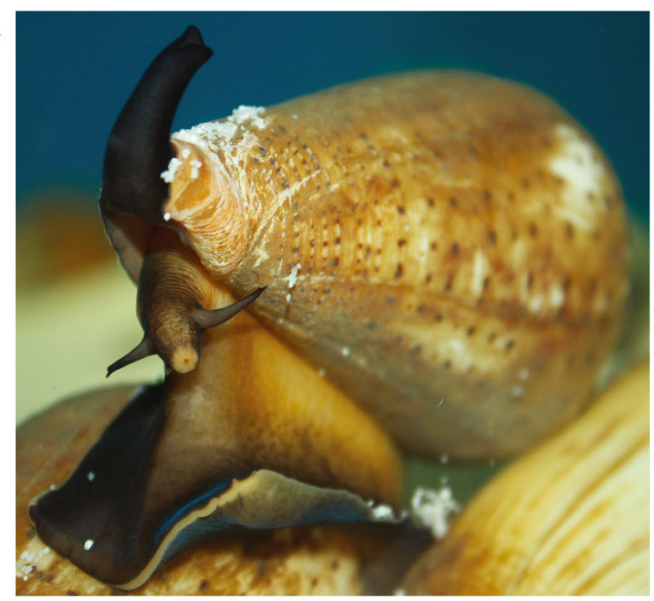

$\mathrm{c}$
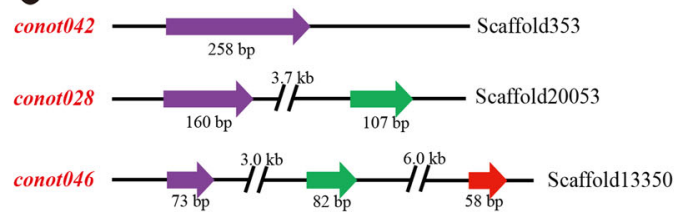

conot128
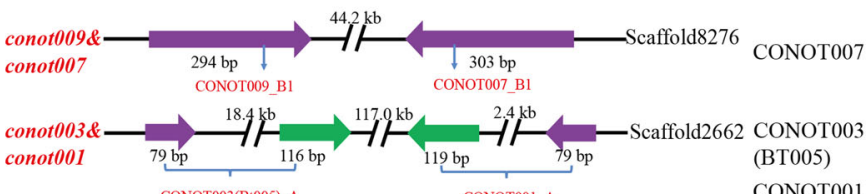

CONOT001

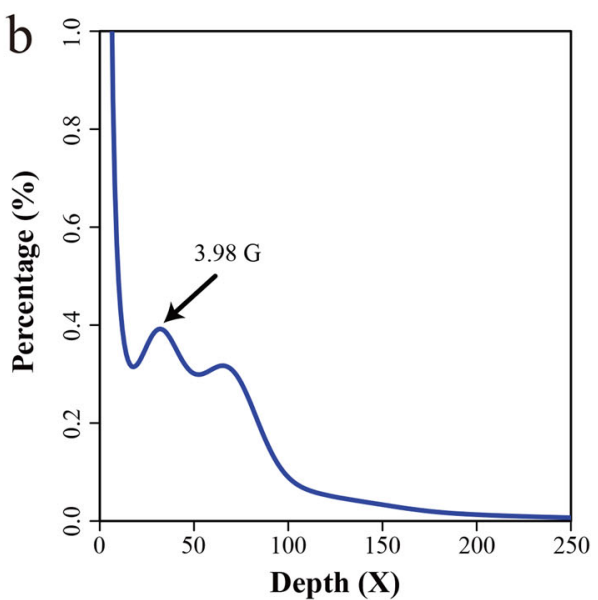

$\mathrm{d}$
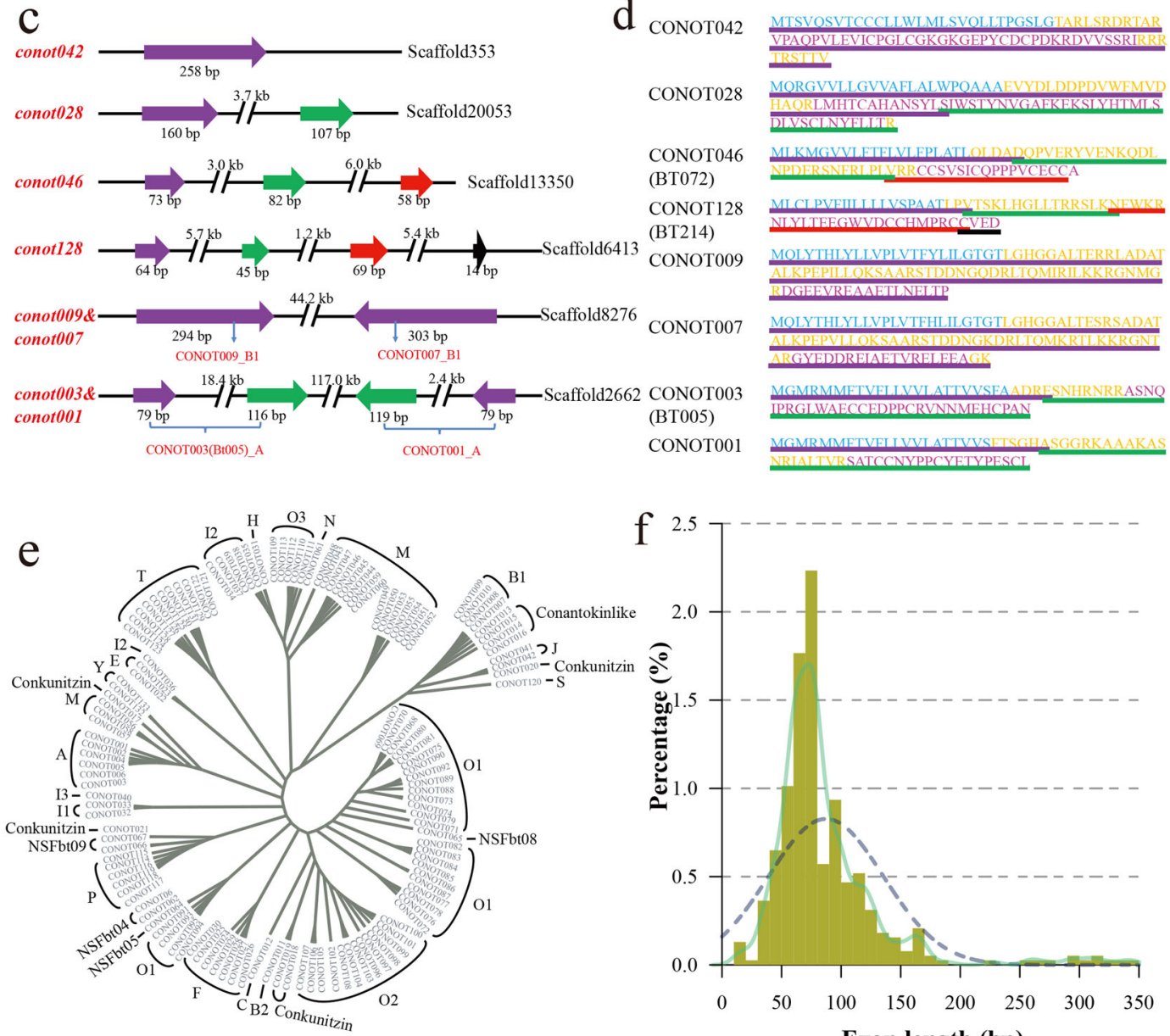

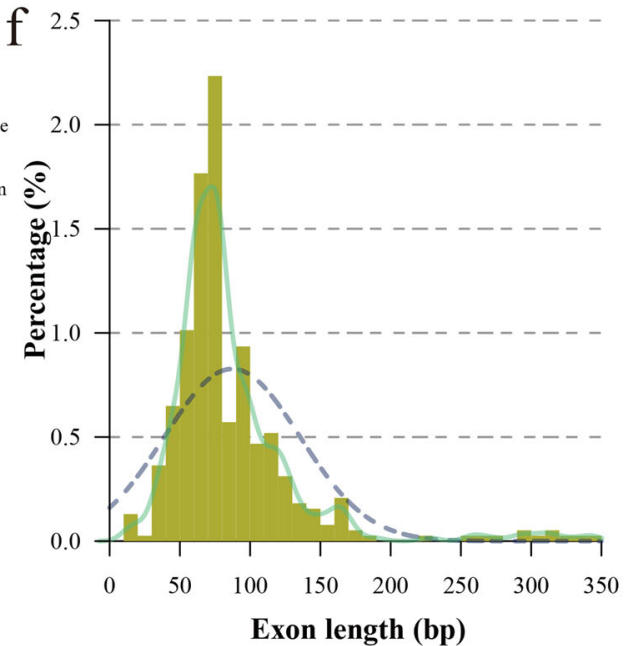

Exon length (bp)

Fig. 1 Identification of 133 conopeptide genes from the assembled genome of $\boldsymbol{C}$. betulinus. a Image of a representative C. betulinus. b The 17mer distribution of sequenced reads. These sequencing data generated from the lllumina short-insert libraries were used for a genome survey analysis ${ }^{8}$. The $x$-axis represents the sequencing depth of each 17-mer, and the $y$-axis represents the percentage of each unique 17-mer. $\mathbf{c}$ Four representative types of exon distribution in the identified full-length conopeptide genes. Regions in purple, green, red, and black stand for the 1st, 2nd, 3rd, and 4th exons, respectively. $\mathbf{d}$ Eight deduced protein sequences of these genes in c. Sequences highlighted in black, yellow, and red are signal peptides, pro-/post-peptides and mature peptides of these precursors, respectively. e Phylogeny of identified conopeptide genes based on predicted signal peptide sequences. $\mathbf{f}$ Detailed distribution of exon length. 
Table 1 Statistics of the genome assembly of $C$. betulinus.

\begin{tabular}{ll}
\hline Genome assembly & Parameter \\
\hline Contig N50 size (bp) & 171,480 \\
Contig number (>100 bp) & 53,852 \\
Scaffold N50 size (bp) & 232,607 \\
Scaffold number (>100 bp) & 41,426 \\
The longest scaffold (bp) & $2,850,889$ \\
Genome GC content & $43.80 \%$ \\
Total length (bp) & $3,430,828,710$ \\
Genome annotation & Parameter \\
Protein-coding gene number & 22,698 \\
Mean transcript length (bp) & $13,448.85$ \\
Mean exons per gene & 7.28 \\
Mean exon length (bp) & 129.69 \\
Mean intron length (bp) & $1,992.04$ \\
\hline
\end{tabular}

and 4 new superfamilies (NSF: NSFbt04, NSFbt05, NSFbt08, and NSFbt09; see Supplementary Table S7).

Fresh muscle tissues were also collected for $\mathrm{Hi}-\mathrm{C}$ sequencing as previously reported ${ }^{18}$. After obtaining the $\mathrm{Hi}-\mathrm{C}$ data, we employed Trimmomatic ${ }^{19}$ to remove adapter sequences and low-quality reads. Subsequently, Juicer $^{20}$ was used to align the clean $\mathrm{Hi}-\mathrm{C}$ reads to the achieved genome assembly for construction of a chromosome-level genome assembly, which is composed of 35 large groups of superscaffolds (Supplementary Figs. S4, S5). Possibly due to the high heterozygosity of Conus genomes (see Fig. 1b), this chromosomal assembly is different from a previous report of 16 pairs of chromosomes in C. magus $^{21}$. However, genome-wide distribution of conopeptide genes is still obvious after matching these gene sequences onto the assembly, since 46 genes were anchored to 38 positions in 16 different groups (see more details in Supplementary Table S8).

\section{A primary genetic central dogma of diverse conopeptides}

The predicted conopeptide genes were aligned to our previously reported 123 conopeptide transcripts from the normalized dataset of a middle body-sized specimen of $C$. betulinus $^{4}$ (Supplementary Table S9). We determined that 127 genes matched to 117 transcripts in the protein sequence alignments (Fig. 2), with parameters set at Query align ratio $\geq 0.80$, Subject align ratio $\geq 0.80$ and identity $\geq 80$ (Supplementary Table S7). When the 133 predicted conopeptide genes were aligned to our previously reported mixture of 215 transcript sequences in $C$. betulinus $^{4}$, a total of 80 potentially corresponding transcripts were determined (Supplementary Table S6); however, few genes (such as conot009 and conot026) may correspond to multiple transcripts individually.

The primary number ratio of entire conopeptide genes to total transcripts is almost 1:1, since a total of 123 transcripts were identified in a similarly middle bodysized snail in our previous report ${ }^{4}$. However, we have also noted that only a fraction $(61 / 133=45.9 \%)$ of the identified conotopeptide genes from the achieved genome assembly are transcribed with transcriptomic evidence (see more details in Supplementary Table S10). Usually, one transcribed conopeptide gene was predicted with sole transcript; however, certain genes (such as conot009) correspond to multiple transcripts, possible due to high intraspecific differences (using different samples for the genome and transcriptome sequencing) or mutationbased variances ${ }^{14}$ (see more detailed explanations under the subsequent section of "Discussion"). When conopeptide superfamilies were considered, we note that the corresponding proportion of transcribed genes was elevated up to $53.1 \%$ per superfamily (Supplementary Table S11).

To investigate the potential relationships among our 215 previously identified conopeptide transcripts from $C$. betulinus $^{4}$, we constructed a phylogenetic tree based on their signal peptide sequences (Fig. 3a). We observed that most of these conopeptides from the same predicted superfamily are clustered together, such as $A, B_{1}$, and $\mathrm{O}_{2}$ superfamilies. However, some predicted superfamilies can not be clustered well possibly due to high sequence diversity. For example, the putative $M$ superfamily was divided into 3 groups except for Bt084 and Bt092. Interestingly, NSFbt03, NSFbt07/NSFbt08, and NSFbt09 were embedded in the $\mathrm{O}_{2}, \mathrm{M}$, and P superfamilies, respectively, indicating their high similarity (although less than 80\%) to each corresponding superfamily.

Meanwhile, we employed two advanced MS systems (TripleTOF 5600 and Q Exactive HF) to perform peptidomics studies (see more details in "Materials and methods") for exploration of various conopeptides in the venom gland of $C$. betulinus. Using deduced protein sequences of the 215 previously identified transcripts as references ${ }^{9}$, we discovered that 1522 peptides, matching 121 conopeptide transcripts, were detected by the Q Exactive HF (Supplementary Table S12) when post-translational modifications (PTMs) were not considered. Similarly, 773 peptides, matching 92 conopeptide transcripts, were identified by the TripleTOF 5600 without consideration of PTMs (Supplementary Table S13). However, only $282(31.8 \%)$ peptides representing 71 (77.2\%) transcripts were identified to be overlapped when using both methods (Fig. 4). By the way, the credibility of conopeptide identification is guaranteed. For example, BT112 is such a short peptide (CFCLTR); however, its peptide spectrum match (PTM) map (Supplementary Fig. S6) proves that the identification is indeed reliable. 

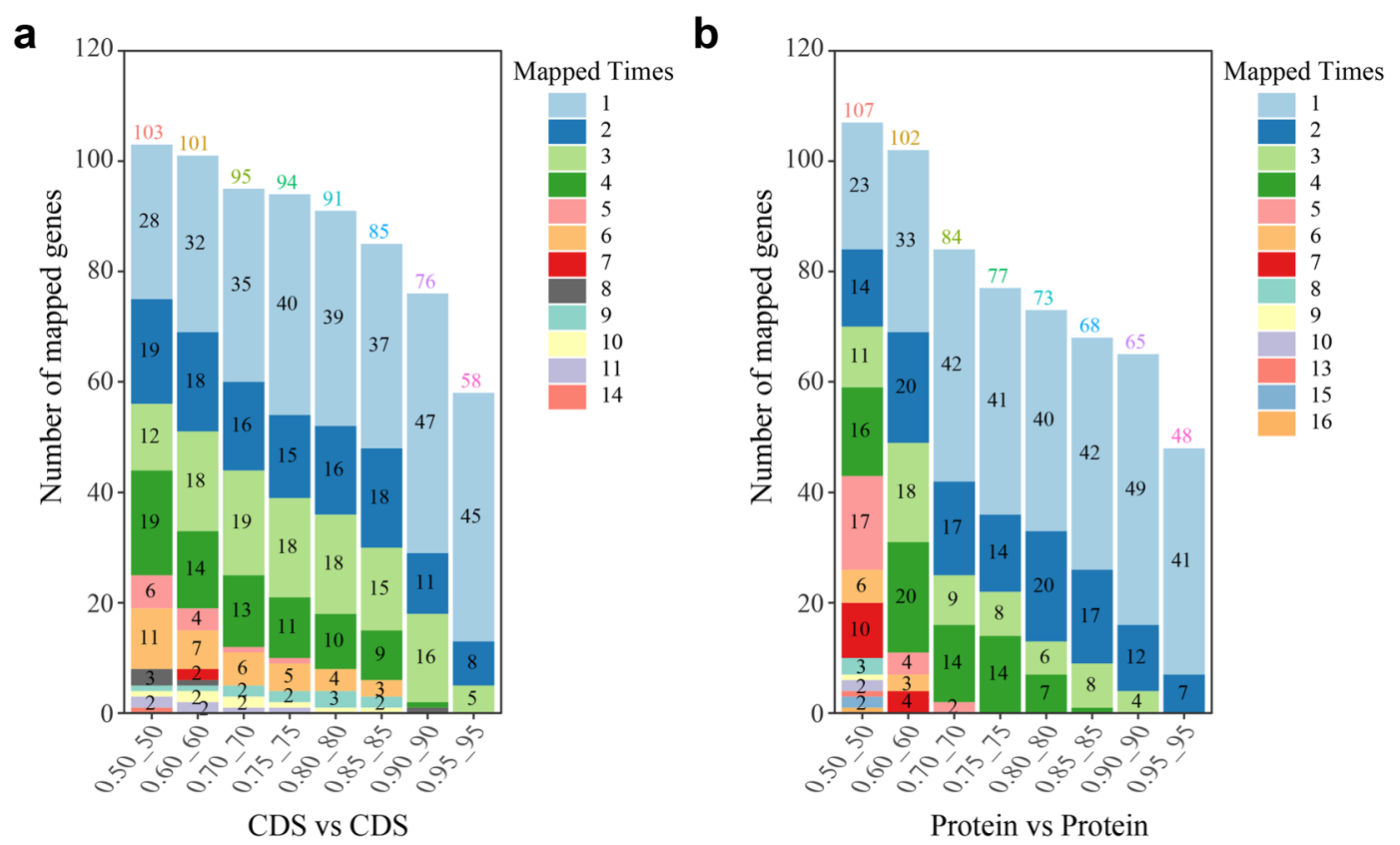

Fig. 2 Sequence alignments of conopeptides from one representative $\boldsymbol{C}$. betulinus sample. We used the coding DNA sequences of conopeptide genes $(\mathbf{a})$ and relevant protein sequences $(\mathbf{b})$ to compare their similarity. The $x$-axis represents the alignment rate and the identity score of each alignment. For instance, 0.50_50 means the alignment rate is 0.50 and the identity score of this alignment is 50 . "Mapped Times" represents the mapped number of single CDS/protein against the reference dataset. The number above each stacked bar stands for the total number of CDS/ protein that exists in the relevant reference dataset. The number within each bar represents the gene number with "Mapped Times". Note that the gene numbers at 1 were not denoted in the figures.

Overall, at least 142 proteins based on 2474 conopeptides with PTMs or 2049 peptide sequences without consideration of PTMs (Supplementary Tables S14, S15, S16) were identified from the two peptidomics methods, suggesting a number ratio of $\sim 1: 10$ s for transcripts or proteins to venom conopeptides. This primary ratio is somehow consistent with a previous report in C. marmoreus $^{7}$, which revealed 2710-6254 peptides using various MS systems (including the high-sensitivity TripleTOF 5600) and matched them to most of the $\sim 100$ transcripts. However, we should realize that different conopeptide proteins may correspond to various numbers of venom peptides (Supplementary Tables S14, S15).

\section{High-throughput predictions for development of potential pharmaceuticals}

The well-known conopeptides $\omega$-MVIIA, AuIB and ImI were first isolated from the venom of corresponding cone snails $^{6,8,22,23}$. The most famous calcium channel blocker $\omega-$ MVIIA (Ziconotide) was approved by the US Food and Drug Administration in $2004^{6}$. AuIB, as one of the 12 reported conopeptides with anti-addictive activity (Supplementary Table S17), specifically inhibits neuronal nicotinic acetylcholine receptors (nAChR; $\alpha 3 \beta 4$ subtype $)^{22}$. ImI has been reported to block nAChRs in fruit fly (Drosophila melanogaster) with an insecticidal activity ${ }^{8,23}$.
Based on the reported peptide sequences of MVIIA, AuIB and ImI (Supplementary Table S18), we searched the 133 conopeptide genes and the 215 transcripts $^{4}$ of $C$. betulinus by using the routine homologous sequence alignment ${ }^{8}$. A total of 13 representative conopeptides were obtained, which ranged from 17 to 28 amino acid residues (aa) in length (Supplementary Table S18). A homologous modeling method ${ }^{8,24}$ was used to predict 3D structures of these homologous conopeptides, confirming their similar stereostructures to MVIIA, AuIB, and ImI, respectively (Fig. 5). Our data suggest that these novel conopeptides may be potentially developed as valuable analgesics, addiction therapies, and insecticides, respectively (Table 2; Supplementary Table S18).

For the homologous sequences of MVIIA (Supplementary Table S18a), these all contain the representative backbone of six cysteines (C) and one glycine (G) (C-G-CCC-C-C; Table $2 \mathrm{a}$ and Fig. 5a). The six cysteine residues are linked by 3 disulphide bonds, serving to stabilize the conformation and to form 4 loops. Similarly, the homologous sequences of AuIB (Supplementary Table S18b) have the representative backbone of 4 cysteines and 2 prolines (P) (CC-P-C-P-C; Table $2 \mathrm{~b}$ and Fig. 5b). The homologous sequences of ImI in Supplementary Table S18c have the representative backbone of CC-P-C-C (Table 2c and Fig. 5c). Four cysteine residues are linked by 
a

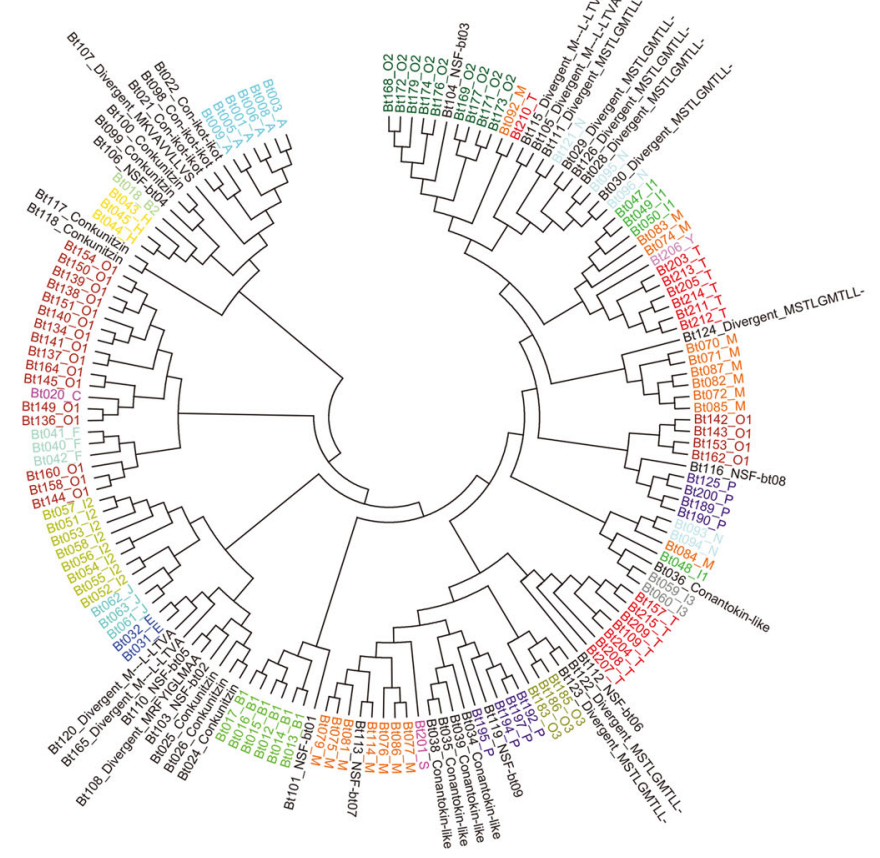

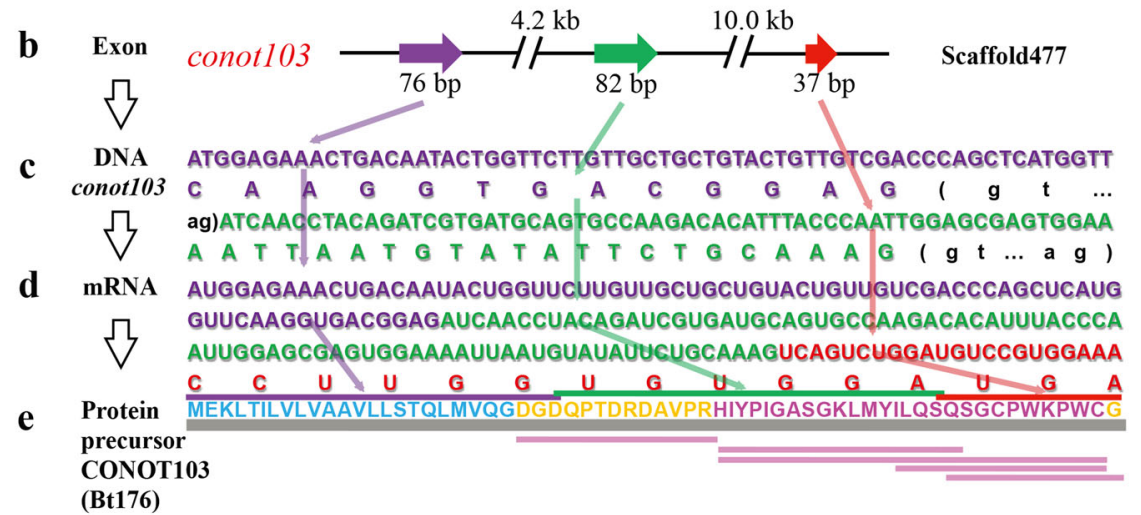

凸

f Protein MEKLTILVLVAAVLLTQLMVQGDGDQPTDRDAVPRHIYPIGASGKLMYILQS̄QSGCPWKPWCG

CONOT103

(Bt176)

Peptides without consideration of PTMs

or post-translational peptides

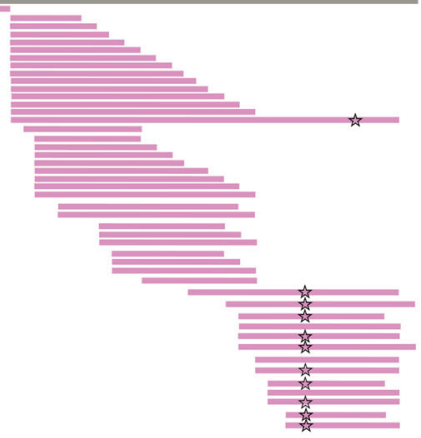

Fig. 3 Identified conopeptide transcripts and post-transcriptional peptides. a Phylogeny of previously deduced conopeptide precursors with full-length signal peptides ${ }^{4} \cdot \mathbf{b}$-e Central dogma of the representative conopeptide CONOT103(Bt176). Regions in purple, green, and red represent the 1st, 2nd, and 3rd exons, or the corresponding sequences, respectively. Sequences highlighted in blue, orange, and red are signal peptides, pro- $/$ post-peptides, and mature peptides of the precursor, respectively. $\mathbf{f}$ Functional conopeptides with post-translational modifications and/or $\mathrm{N}$ - and Cterminal truncations ${ }^{7}$ that were detected by MS. Amino acid sequences with open star illustrate amino acid modifications at hydroxy-prolines (P). 

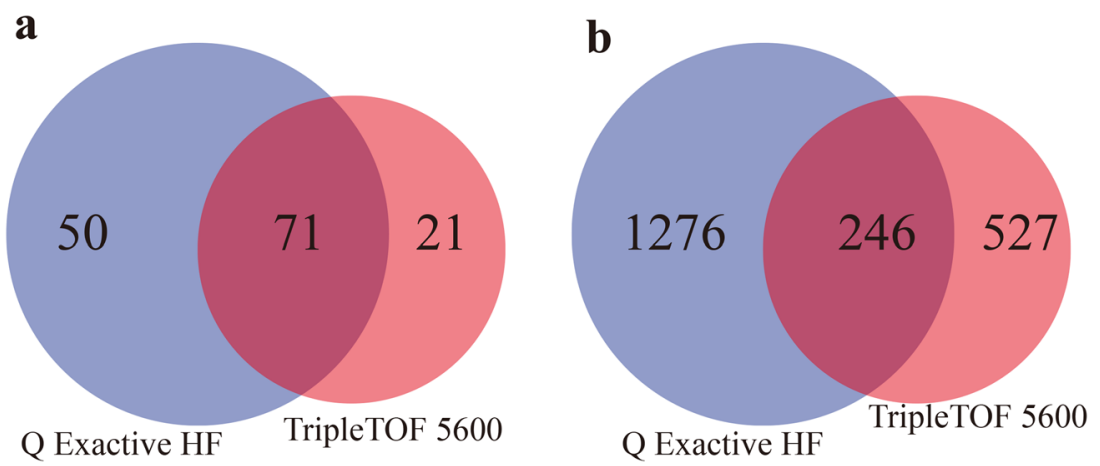

Fig. 4 Analysis of peptidomics data of $C$. betulinus venom by two different mass spectrometry (MS) systems. a Numbers of identified proteins. $\mathbf{b}$ Numbers of identified peptides.

2 pairs of disulphide bonds, in which loop 1 (between the 1st and the 3rd cysteines) and loop 2 (between the 2nd and the 4th cysteines) are variable to determine the selectivity of corresponding nAChR subtypes ${ }^{1,8}$.

\section{Discussion}

Next-generation high-throughput sequencing technologies have opened up new opportunities for genome-wide studies on various animals, plants, and bacteria. In the present study, we combined Illumina short reads and PacBio long reads to generate the first Conus genome assembly, which revealed a primary genetic central dogma of conopeptides in the representative C. betulinus.

In fact, an early attempt to sequence $C$. bullataus genome by Olivera and colleagues ${ }^{13}$ was published in 2011. Although this is a trial shotgun survey with low quality, the authors provided many detailed characterizations. For example, they randomly selected 30 million genomic reads and determined the GC content to be $42.88 \%$, which is similar to our genome-wide calculation of $43.80 \%$ for the C. betulinus genome (Supplementary Fig. S1). Using genomic and transcriptomic reads, the authors had developed a novel method to estimate genome $\operatorname{size}^{13}$; the final estimate for the size of $C$. bullataus genome was $2.56 \mathrm{~Gb}$, which is less than our assembly $(3.43 \mathrm{~Gb})$ and estimate $(3.99 \mathrm{~Gb})$ for the C. betulinus genome. This difference also supports the high diversity of various Conus genomes.

Recently, Phuong and Mahardika ${ }^{14}$ successfully recovered venom gene superfamilies from the genomes of 32 cone snails by using targeted sequencing techniques. They found that conopeptide gene superfamilies are composed of 1-6 exons (covering our present report of 1-4 exons for C. betulinus; see more representative details in Fig. 1c), and single exon is typically short in length (5-444 bp; covering our current range of 14-355 bp for C. betulinus) with an average length of $85 \mathrm{bp}$ (similar to the average of $87 \mathrm{bp}$ for C. betulinus; see Fig. 1f). Interestingly, the

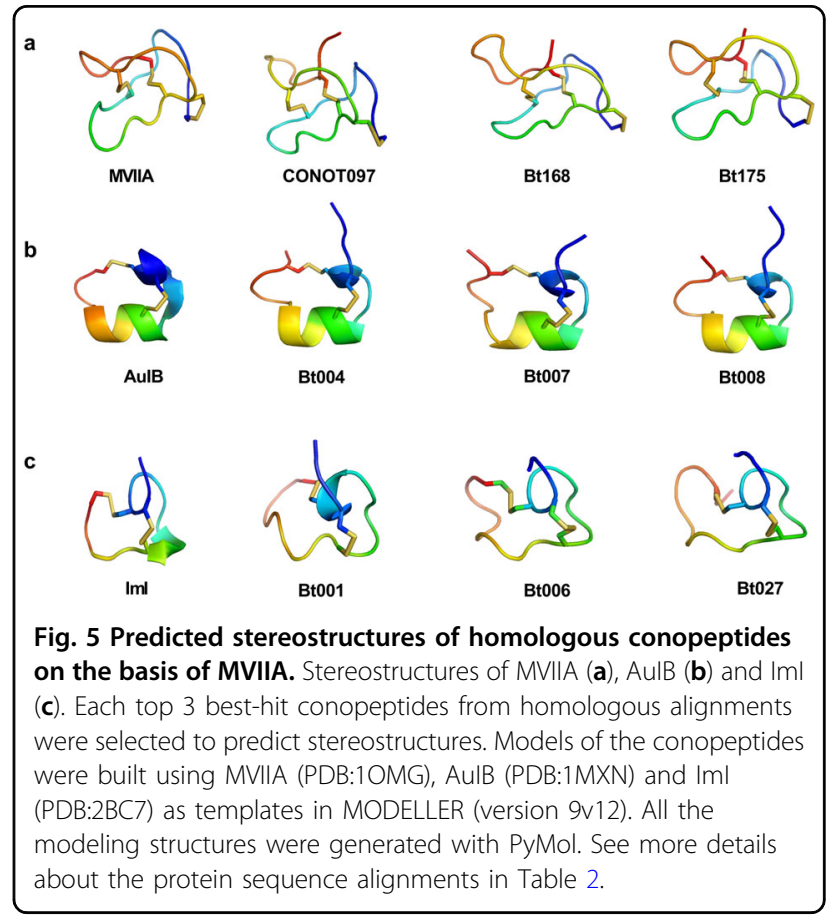

detailed exon length distribution from the big dataset of 32 Conus species is almost the same as our data in Fig. 1f, providing a solid support to our present high-quality genome assembly for $C$. betulinus. This recent work also improved our understanding of conopeptide molecular evolution. Based on the extracted genomic data, the authors identified variable conotoxin gene copies from 120 (C. papilliferus) to 859 (C. coronatus), which cover our present prediction of 133 conotoxin genes in the $C$. betulinus genome. Although they stated that diet specificity did not predict patterns of conopeptide evolution, we observed a primary evolutionary pattern for those species within the same phylogenetic clade. It somehow seems to be true that a primitive Conus species usually owns less conopeptide gene copies (see more details in the 
Table 2 Protein sequence alignments of the representative conopeptides.

\begin{tabular}{|c|c|c|}
\hline Name & Conotoxin sequence & GenBank accession no. \\
\hline \multicolumn{3}{|c|}{ (a) Analgesic activity } \\
\hline MVIIA & YDCCTGSCRS - -GKC * - - & P05484.2 \\
\hline CONOT097 & SQCCSSFCRRRMGKCYY- & --- (Present study) \\
\hline Bt168 & -YSTQCCSTYCRHRVHKCA - - & KU563979.1 \\
\hline Bt175 & YSTQCCTTYCRR--HKCA- - & KU564050.1 \\
\hline \multicolumn{3}{|c|}{ (b) Antiaddictive activity } \\
\hline AulB & & P56640.2 \\
\hline Bt007 & & KU564009.1 \\
\hline Bt004 & YETYPESCL - & KU564008.1 \\
\hline Bt008 & YPPCYEAYPEICL- & KU317629.1 \\
\hline \multicolumn{3}{|c|}{ (c) Insecticidal activity } \\
\hline Iml & & KJ801971.1 \\
\hline Bt001 & & KU563886.1 \\
\hline Bt006 & -RGCCSHPACSVNHPELC- - & KU563888.1 \\
\hline Bt027 & -DDCCPDPACRQNHPELCSS & KU564013.1 \\
\hline
\end{tabular}

The list is simplified from Supplementary Table S18 for a 3D-structural comparison in Fig. 5.

Note: Conserved residues (with similar properties) among different conopeptides are highlighted in the same background color. *, Amidated C-terminus.

Fig. 5 of Phuong and Mahardika ${ }^{14}$ ), which is consistent with the low gene copy number (133) and the proposed primitive status ${ }^{2}$ for the vermivorous $C$. betulinus.

As reported previously ${ }^{14,25}$, only a fraction of conopeptide genes in Conus genomes were proved to be transcribed when compared transcriptome and genome sequences. In our present study, the entire gene number (133) is slightly higher than the sum of identified transcripts $(\sim 123)$ in individual cone snail. By comparing the transcriptome data to the 133 predicted conopeptide genes, we figured out how many genes transcribed in the mixed and individual samples, respectively. Our results showed that 87 (65.4\%) conopeptide genes are transcribed in the pooled samples (Supplementary Table S6). The proportion of conopeptide genes transcribed per gene superfamily was up to $72.5 \%$ (Supplementary Table S11). However, in the individual middle-sized cone snail, only 61 (45.9\%) conopeptide genes are transcribed with transcriptomic evidence (Supplementary Table S10). Such transcription pattern was also comparable to the previously reported proportions of $24 \%-63 \%$ from 32 various Conus species ${ }^{14}$, confirming that only a fraction of conopeptide genes are transcribed. This discrepancy in genome and transcriptome data for conopeptides may be caused by variable parameters in life stage and geographic localization, or by the genome itself since many genes are no longer functional (i.e., pseudogenized), or by different specimens or mutation-based variances ${ }^{14}$. We may be able to improve the present version of genome assembly with more PacBio and $\mathrm{Hi}-\mathrm{C}$ sequencing reads; in this case, few more conopepetide genes are expected to be identified, which can increase the align number to corresponding transcripts.

Dramatic interspecies and even intraspecies variations have been reported previously ${ }^{9,11,26-28}$. Milked venom has been thought to be perfect for subsequent MS examination, since it lacks cellular debris and unprocessed toxins $^{9,26}$; however, production of different venoms by same individual was also revealed as an unsuspected contribution to venom diversity ${ }^{26}$. In the present study, however, we had to apply the routine and conveniently dissected venoms, which may have complicated the component profile of venom conopeptides. High diversity in conopeptide transcripts were also extensively documented in various Conus species ${ }^{9,27,28}$, with a big range from 100 up to 522 (or more) ${ }^{9}$ that covers our individual 123 transcripts for $C$. betulinus. These accumulated data indicate that the number ratios of total conopetpide genes: transcripts: peptides in various cone snails may present somewhat variances. Therefore, our present ratio for $C$. betulinus may be temporarily special. More studies and samples are required to confirm these primary data, although they may be relatively stable in this species.

Recent estimations of venom conopeptide number at the proteomic level generate an increasing value to several thousands ${ }^{9}$ using highly sensitive MS systems. Such 
discrepancy between transcriptomic and proteomic data in terms of sequence could be explained not only by posttranslational process ${ }^{9}$, but also by degenerated products ${ }^{27}$ (although difficult for determination). As reported in detail by Dutertre et al. ${ }^{9}$, variable peptide processing at the proteomic level, generating a big diversity of conopeptides with alternative cleavage sites, post-translational modifications, and $\mathrm{N}$-/C-terminal truncations, may explain how a limited set of $\sim 100$ transcripts can generate thousands of conopeptides in the venom of individual cone snail.

In the present study, additional peptide diversity was often created by PTMs $^{9}$ (Supplementary Tables S13, S14; not easily be predicted from precursor sequences), such as at 4-hydroxy-proline $(\mathrm{P})$, 5-hydroxy-lysine (K), gammacarboxyglutamic acid (E), and pyroglutamic acid (E). According to the total modifications of these detected peptides (Supplementary Table S14), we estimate that the maximal number of conopeptides may reach 6653 . Interestingly, conopeptide Bt018 may have the maximal (up to 4608) modified combinations because it matches to a higher number of peptides (225) and involves more modifications (4 different modifications at 11 amino acid sites, and some sites with different types of modification; Supplementary Table S16). Related protein coverage areas, number of identified peptides, and modification sites of the representative conopeptide Bt018 are summarized in Fig. 6. In summary, taking the number of conopeptide genes identified in the genome assembly into consideration, we assume that the number ratio of genes: transcripts: precursors: post-translationally modified peptides of conopeptides is around 1:1:1:10s. As stated in a previous report ${ }^{9}$, the vast majority of detected conopeptides are generated from a more limited set of genes/ precursors through peptide processing, which produces various conopeptides with alternative cleavage sites, posttranslational modifications, and highly variable $\mathrm{N}-/ \mathrm{C}$ terminal truncations (see the representative Fig. 3f). This variable peptide processing, along with potentially intraspecies variation ${ }^{4,9}$, may explain how 133 genes (Supplementary Table S6) and 123 transcripts (Supplementary Table S7) can generate thousands of conopeptides in the venom of individual cone snail.

Recovery of intact conopeptide proteins from the detected peptides prompted us to look back into the assembled genome and to search for a complete central dogma of conopeptides. Here, we illuminated 9 representative conopeptides, such as CONOT006(Bt003), CONOT019(Bt025), CONOT039(Bt057), CONOT046 (Bt072), CONOT52(Bt075), CONOT055(Bt077), CON OT071(Bt150), CONOT103 (Bt176) and CONOT129 (Bt207), with both full-length exons and complete mature peptides as examples. In fact, Bt176 was encoded by the conot103 gene, which is localized on the Scaffold 477 with

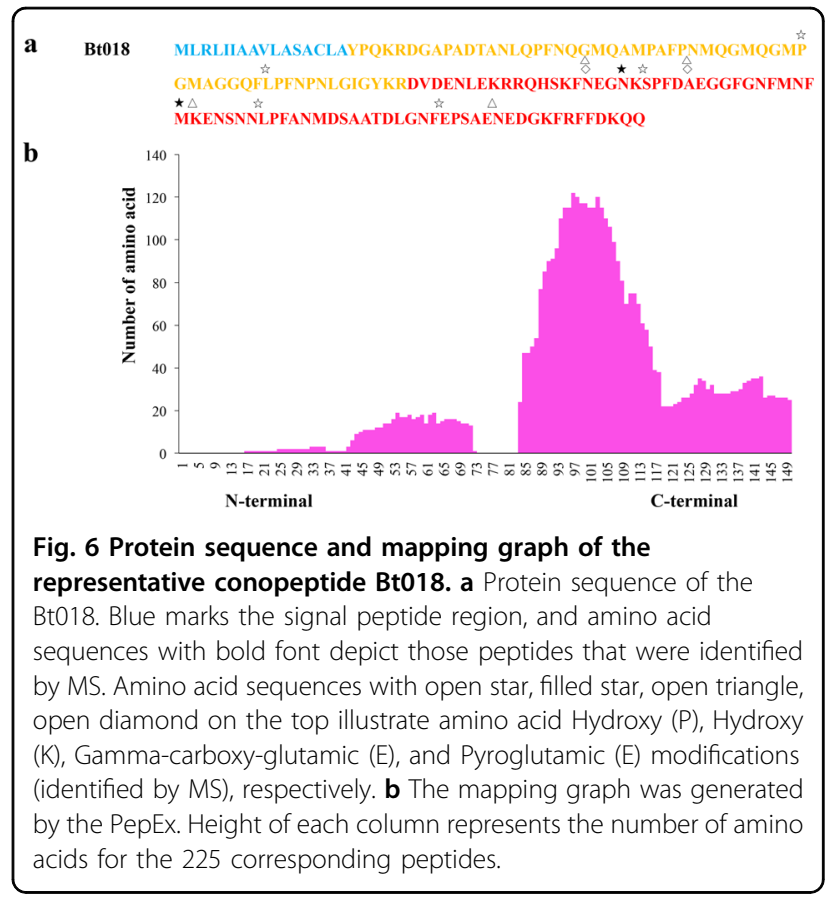

3 exons (Fig. 3b). The transcribed mRNA is $195 \mathrm{bp}$ in length, with a stop codon (UGA) at the end. The full length of deduced precursor consists of 64 aa, in which 1 -26th, 26-53rd, and 53-64th aa are encoded by the exons 1,2 , and 3, respectively (Fig. 3b-d). Meanwhile, the pro-peptide (24-36th aa), mature peptide (37-63rd aa), and post-peptide (64th aa) have also been detected by the 2 peptidomics methods (Fig. 3e). However, some conopeptides can only be detected in the mature peptide regions (Fig. 7a; such as Bt050), whereas some signal peptide regions can also be detected by the peptidomics sequencing (Fig. 7b; such as Bt053). Meanwhile, Bt018 and CONOT103(Bt176) were detected with a total of 225 and 46 post-translationally modified peptides, respectively; their high transcription levels, revealed in our previous report ${ }^{4}$ (Supplementary Table S19), concurrently support their critical importance in prey capture and/or defense ${ }^{29,30}$.

As discussed in previous reports ${ }^{9}$, the high sensitivity of the TripleTOF system allowed fine characterization of many peptide variants for each conopeptide transcript. However, most of the peptide diversity corresponded to truncated forms of the mature peptide or pro-peptide sequences. It is interesting to observe that conopeptide proteins tend to be digested at several favorite cleavage sites (Fig. 8). We used CONOT019(Bt025) as an example to examine which enzymes and what kind of cleavage sites $^{31}$ are preferred in a precursor. Our results proved that there are 35 possible cleavage sites in this protein sequence, which could be digested by 18 different enzymes (Supplementary Table S20). The enzyme with 

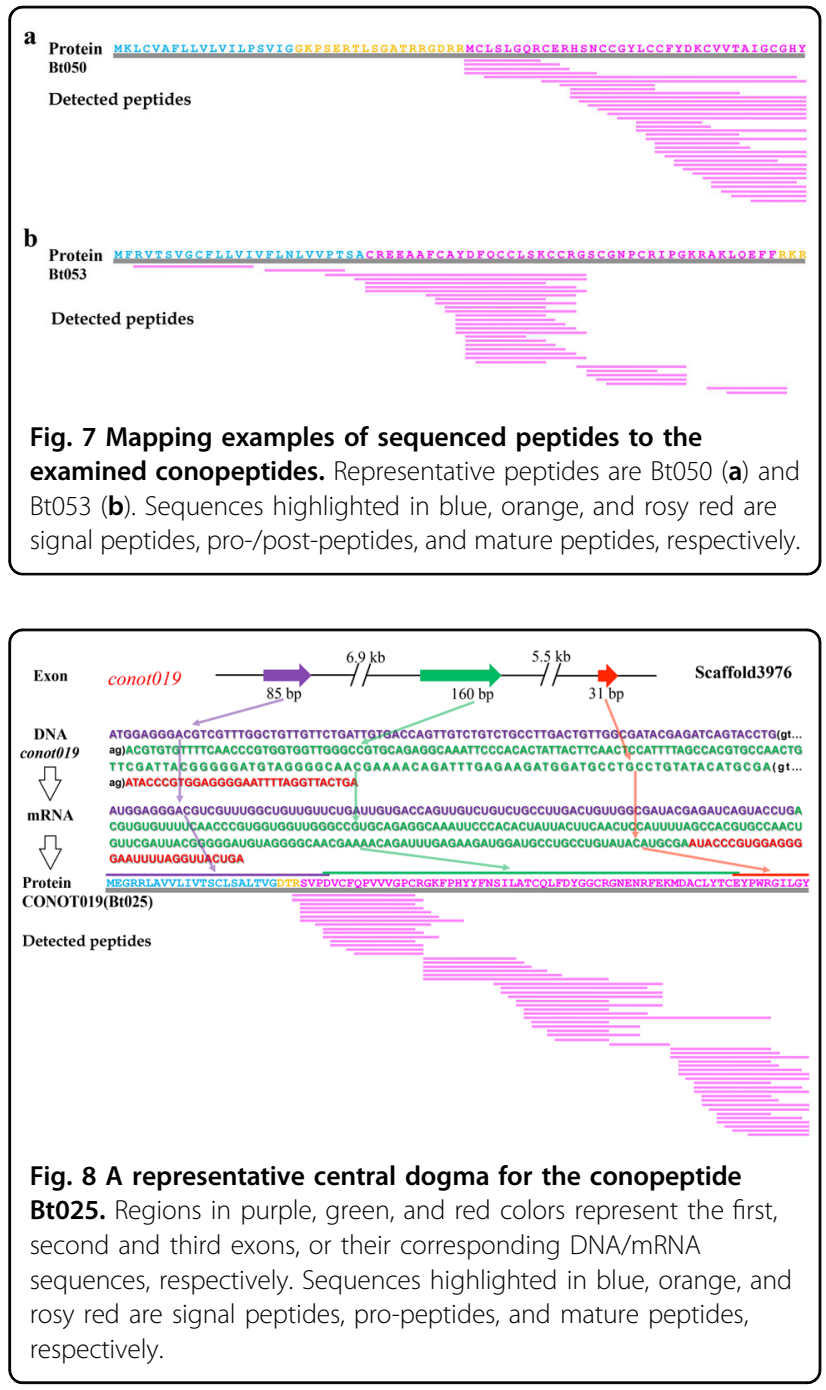

the most cleavage sites $(n=15)$ is proteinase $K$, which preferentially cleaves aa residues ${ }^{18}$ of A, E, F, I, L, T, V, W or Y. However, the $41^{\text {st }} \mathrm{R}$ of the representative $\mathrm{Bt} 025$ is the most cleaved site (with 12 detected peptides; see Supplementary Table S20) that could be digested by 3 various enzymes, including Arg-C proteinase, clostripain and trypsin.

In summary, this is the first report of a Conus genome assembly. With the integration of multi-omics (genomics, transcriptomics and peptidomics) data, we attempted to illustrate the special genetic central dogma of conopeptides in C. betulinus, assuming that the primary number ratio for total conopeptide genes: transcripts: proteins: post-translationally modified peptides is $\sim 1: 1: 1: 10$ s. Some predicted conopeptides in our present study are similar to the well-known analgesic $\omega$-MVIIA, addiction therapy AuIB and insecticide ImI in cysteine backbone and 3D structure, suggesting that these novel peptides could be developed as pharmaceutical candidates.

\section{Materials and methods}

\section{Genome sequencing and assembly}

Specimens of wild C. betulinus, 8-9 cm in length, were collected from offshore areas of Sanya City, Hainan Province, China. We employed two different strategies to sequence the genome of this popular Conus species in the South China Sea.

\section{Illumina sequencing}

Genomic DNA was extracted from the pooled muscle of two specimens using Qiagen GenomicTip100 (Qiagen, Gaithersburg, MD, USA). In accordance with the standard protocol from Illumina (San Diego, CA, USA), we constructed three short-insert libraries $(250,500$, and $800 \mathrm{bp})$ and four long-insert libraries $(2,5,10$, and $20 \mathrm{~kb})$ for sequencing on an Illumina HiSeq 2000 system $^{15}$. In total, we generated $315.24 \mathrm{~Gb}$ of raw reads (Supplementary Table S1). SOAPfilter v2.2 ${ }^{32}$ with default parameters was employed to collect clean data by removal of low-quality reads (including reads with 10 or more nonsequenced/ low-quality bases), PCR duplicates and adapter sequences.

\section{PacBio sequencing}

Genomic DNA was extracted from the muscle of another specimen for construction of a 20-kb PacBio SMRT libraries, which were sequenced by a PacBio Bioscience Sequel platform (Menlo Park, CA, USA). Finally, we obtained a total of $239.69 \mathrm{~Gb}$ of long reads (Supplementary Table S2).

\section{Genome assembly}

We estimated the genome size to be $3.98 \mathrm{~Gb}$ (Fig. 1b; Supplementary Table S3) on the basis of the 17-mer depth frequency distribution method ${ }^{15}$. A routine hybrid strategy $^{16}$ was applied to assemble the genome sequences. First, SparseAssembler ${ }^{33}$ with optimized parameters (K 51 NodeCovTh 2 EdgeCovTh 1) was performed to generate a De Bruijin graph based original contigs by using the Illumina short-insert reads. Subsequently, we employed the DBG2OLC ${ }^{34}$ program to remap these contigs upon the PacBio corrected long reads for construction of longer contigs. We then used Minimap v2 ${ }^{35}$ and Racon v1.3.1 $1^{36}$ to generate consensus contigs. Finally, we employed SSPACE_Standard v3.0 $0^{37}$ and SSPACE-LongRead v1-1 ${ }^{38}$ to obtain a more continuous assembly.

\section{Genome annotation}

Homology-based predictions were performed to detect known transposable elements (TEs) by running RepeatMasker (version 3.3.0) ${ }^{39}$ against the RepBase TE library (release 17.01) ${ }^{40}$. Repeat sequences at DNA and protein levels were identified using the TE library and RepeatProteinMask ${ }^{39}$. We constructed de novo repeat library using LTR_FINDER ${ }^{41}$ and RepeatModeler ${ }^{39}$ 
(version 1.0.5), and then used RepeatMasker ${ }^{39}$ to obtain TE results with detailed classifications for each repeat family.

Tandem repeats were also searched in the archieved genome assembly using Tandem Repeats Finder (TRF, version 4.04$)^{42}$. All the repeat sequences were finally combined with non-redundant repetitive sequences (Supplementary Table S4). Protein coding genes were predicted by combination of de novo, homology, and transcriptome ${ }^{4}$ based methods ${ }^{15}$. All the predicted genes (Supplementary Table S5) were integrated into a comprehensive and non-redundant gene set using Maker$2.31 .8^{43}$.

\section{Genome assembly evaluation}

We evaluated the completeness ${ }^{15}$ of our C. betulinus assembly with metazoan and eukaryotic benchmarking universal single-copy orthologs (BUSCOs v3.0 ${ }^{17}$ ).

\section{Screening of conopeptide genes from the assembled genome Identification of conopeptide genes}

A total of 215 conopeptide transcripts were previously identified by us from 6 transcriptome datasets of $C$. betulinus ${ }^{4}$. Corresponding cDNA sequences were mapped against our present genome assembly using Blast ${ }^{44}$ and Genewise $^{45}$. Subsequently, the alignment sequences of scaffolds were extracted using a Perl script based on high similarity.

With reference to the corresponding transcripts, these sequences were divided into exon and intron regions (Supplementary Table S6) to construct conopeptide/ conotoxin genes (named as conot001-conot133) manually. These predicted genes were then translated into corresponding proteins (precursors) and named as "CONOT001-CONOT133" (Supplementary Table S7). Proteins of the same genes and transcripts are termed in the format of 'CONOTx + (Bty)' ( $\mathrm{x}$ and $\mathrm{y}$ refer to Arabic number suffixes), such as CONOT103(Bt176).

Based on at least $75 \%$ identity in the conserved signal peptide sequences ${ }^{10,46}$, these translated protein sequences were assigned to various conopeptide superfamilies and groups ${ }^{4}$.

\section{Phylogenetic analysis of the identified conopeptides}

Complete signal peptide sequences of the 133 genes and 215 transcripts were manually selected. They were then aligned online using Multalin ${ }^{47}$ (v 5.4.1). Phylogenetic trees were constructed by using MEGA $6^{48}$ with the maximum likelihood method. Statistical supports were assessed with 1000 bootstrap pseudo-replicates, and those classic superfamilies were marked with different colors (Fig. 1e).

\section{Gene and transcript analysis}

The predicted 133 genes (conot001-conot133) were realigned onto the 215 transcripts ${ }^{4}$ using BLAST with an E-value $<1.0 \mathrm{e}^{-5}$ and an alignment rate $>0.6$. Solar v0.9.6 ${ }^{49}$ was conducted to link high-identity segment pairs. Those low-quality sequences with alignment rate $<0.5$ and mapping identity $<0.5$ were discarded.

To characterize the detailed proportion of transcribed conopeptide genes, we mapped nucleotide sequences of the totally identified 215 transcripts $^{4}$ to the CDS of 133 conopeptide genes by restricting the blast alignment ratio $\geq 95 \%$ and the sequence identity $\geq 95 \%$. These settings of such stringent cutoffs were due to the high conservation of signal peptides and the good similarity of conopeptide genes within a superfamily ${ }^{14}$. We also calculated the number of transcribed conopeptide genes in the middle body-sized snail (with 123 identified transcripts), which potentially represents the transcription pattern in single snail.

\section{Hi-C sequencing and assembly Hi-C library construction}

Fresh muscle tissues were collected for the $\mathrm{Hi}-\mathrm{C}$ sequencing as described in the previous studies ${ }^{18}$. In brief, $\sim 1 \mathrm{~g}$ of muscle sample was cross-linked with $1 \%$ formaldehyde for $10 \mathrm{~min}$ at room temperature and quenched with glycine (final concentration of $0.2 \mathrm{M}$ ) for $5 \mathrm{~min}$. The cross-linked cells were subsequently lysed to extract nuclei. The DNAs in nuclei were further digested with $\mathrm{MboI}$ and marked with biotin-14-dCTP (Invitrogen, Carlsbad, CA, USA) and then ligated by T4 DNA ligase. After reversing cross-links, the ligated DNA was sheared to 300- to 500-bp fragments. The DNA fragments were purified through biotin-streptavidin mediated pull-down and were further blunt-end repaired and A-tailed using NEBNext Ultra II DNA library Prep Kit (New England Biolabs, Ipswitch, MA, USA) according to manufacturers' instructions. Finally, the $\mathrm{Hi}-\mathrm{C}$ libraries were quantified and sequenced on the Illumina HiSeq X Ten platform (San Diego, CA, USA) with the 150 paired-end mode.

\section{Hi-C Proximity-guided assembly}

After obtaining Hi-C reads, we employed Trimmomatic (version 0.38$)^{19}$ to remove the adapter sequences and lowquality reads. Then, Juicer v1.6. $2^{20}$ was used to align $\mathrm{Hi}-\mathrm{C}$ reads to the achieved genome to obtain the connection relationship between contigs, and $3 \mathrm{~d}$-dna (version 180922$)^{50}$ was used to scaffold those contigs through the misjoin correction algorithm, the scaffolding algorithm, and the merging algorithm using default parameters. The chromosome-level genome assembly was achieved after polishing, sealing, and chromosome splitting of mega-scaffolds. Finally, misassembled contigs in visual were corrected using JucieBox (v 1.8.8) ${ }^{51}$. Align the 
identified 133 gene sequences to the 35 large groups using blastn (version 2.7.1+) with the following parameters: blastn -query gene.fasta -db all_group.fasta -outfmt 5 -out xx.out -evalue $1 \mathrm{e}^{-5}$.

\section{Peptidomics sequencing to identify venom conopeptides Venom collection}

Venom ducts of collected C. betulinus $(n=3)$ were dissected quickly on ice for subsequent homogenization ${ }^{52}$ in $30 \%$ acetonitrile $(\mathrm{ACN})$ containing $0.1 \%$ trifluoroacetic acid (TFA) $)^{53,54}$. Protease inhibitor cocktail (Roche, Shanghai, China) was added to prevent degradation of proteins and peptides according to the manufacturer's instructions ${ }^{55}$. After centrifugation of $16,100 \times g$ at $4{ }^{\circ} \mathrm{C}$ for $30 \mathrm{~min}$, the supernatant (crude venom) was collected for lyophilization and final storage at $-20{ }^{\circ} \mathrm{C}$ until use.

\section{Preparation of venom samples}

Crude venom was suspended in $0.1 \mathrm{M}$ Tris- $\mathrm{HCl}(\mathrm{pH}$ 8.5) with $8 \mathrm{M}$ urea. Protein concentrations were measured using the standard Bradford method. Denatured proteins and peptides were reduced with $10 \mathrm{mM}$ dithiothreitol (DTT) at $56^{\circ} \mathrm{C}$ for $45 \mathrm{~min}$. After cooling to room temperature, the solutions were alkylated with $55 \mathrm{mM}$ Iodoacetamide (IAA) in dark at room temperature for $30 \mathrm{~min}$. Venom peptides were extracted and purified using the Strata-X C18 column (Phenomenex Inc., Torrance, CA, USA) according to the manufacturer's instructions. The final eluates (venom peptides) were dried in a ScanVac freeze drier (LaboGene, Lynge, Denmark) for storage at $-20^{\circ} \mathrm{C}$ before use. Venom peptides were quantified using a NanoDrop A280 system $^{56}$ (Thermo Fisher Scientific, Waltham, MA, USA).

\section{Peptide fractionation}

Two different HPLC approaches were applied, including strong cation exchange (SCX) and high-pH reverse phase (Hp-RP) chromatography. In the Hp-RP procedure, $100 \mu \mathrm{g}$ of venom peptides was separated by a Gemini Hp$\mathrm{RP}$ column $(4.6 \times 250 \mathrm{~mm}, 5 \mu \mathrm{m}, 110 \AA$; Phenomenex Inc.). Fractionation was performed using a linear gradient of $0 \%-40 \%$ of buffer A ( $80 \%$ ACN, $20 \mathrm{mM} \mathrm{NH}_{4} \mathrm{FA}, \mathrm{pH}$ 10) at a flow rate of $1 \mathrm{~mL} / \mathrm{min}$ for $40 \mathrm{~min}, 40 \%-90 \%$ of buffer A for $2 \mathrm{~min}$, and $90 \%$ of buffer A for $3 \mathrm{~min}$. In the SCX HPLC procedure, $100 \mu \mathrm{g}$ of venom peptides was separated by a Luna SCX column $(4.6 \times 250 \mathrm{~mm}, 5 \mu \mathrm{m}$, $110 \AA$ A; Phenomenex Inc.). Fractionation was performed using a linear gradient of $0 \%-40 \%$ of buffer $\mathrm{B}(25 \% \mathrm{ACN}$, $1 \mathrm{M} \mathrm{KCl}, 10 \mathrm{mM} \mathrm{KH}_{2} \mathrm{PO}_{4}, \mathrm{pH} 3.0$ ) at a flow rate of $1 \mathrm{~mL} /$ min for $40 \mathrm{~min}, 40 \%-90 \%$ of buffer B for $2 \mathrm{~min}$, and $90 \%$ of buffer B for $3 \mathrm{~min}$. All the HPLC procedures were manipulated in a 20AD HPLC system (Shimadzu, Kyoto, Japan). Absorbance was monitored at $214 \mathrm{~nm}$, and the fractions were collected along the gradient for lyophilization.

\section{Liquid chromatography-tandem MS (LC-MS/MS) Analysis} TripleTOF 5600 LC-MS/MS was performed using a TripleTOF 5600 MS System (AB Sciex, Foster City, CA, USA) coupled to a nano-HPLC system (Shimadzu). The peptides of each fraction from the SCX were separated by nano-HPLC on an in-house packed $12 \mathrm{~cm} \times 75 \mu \mathrm{m}$ Ultimate XB-C18 column ( $3 \mu \mathrm{m}, 120 \AA \AA$; Welch Materials Inc., Shanghai, China) at a flow rate of $300 \mathrm{~nL} / \mathrm{min}$. Each fraction was loaded in buffer $\mathrm{C}(5 \% \mathrm{ACN}, 0.1 \%$ formic acid (FA)) and eluted with a linear 40-min gradient of $5 \%-45 \%$ buffer D (95\% ACN, 0.1\% FA). MS parameters were set as follows: electrospray voltage of $2.5 \mathrm{kV}$, positive ion data-dependent scan mode, full scan range of $350-1500 \mathrm{~m} / \mathrm{z}$, selection of the top 30 ions, and dynamic exclusion duration $18 \mathrm{~s}$.

Q Exactive HF Another LC-MS/MS was performed using a Q Exactive HF coupled to an UltiMate 3000 UHPLC system (Thermo Scientific). The peptides of each fraction from the Hp-RP were separated by a $75 \mu \mathrm{m} \times$ $25 \mathrm{~cm}$ in-house analytical column that packed with Ultimate LP-C18 particles ( $3 \mu \mathrm{m}, 120 \AA$; Materials Inc.) at a flow rate of $300 \mathrm{~nL} / \mathrm{min}$. Each fraction was loaded on a trap column $(30 \mu \mathrm{m} \times 5 \mathrm{~mm}, \mu$-Precolumn; Thermo Fisher Scientific) with buffer E ( $2 \%$ ACN, $0.1 \%$ FA) in $5 \mathrm{~min}$, followed by a linear 40 -min gradient of $5 \%-35 \%$ buffer F ( $98 \% \mathrm{ACN}, 0.1 \% \mathrm{FA})$, and then increased to $80 \%$ in $5 \mathrm{~min}$. Mass spectrometry data were acquired with a top30 data-dependent mode scan method. The electrospray voltage was set to $1.6 \mathrm{kV}$ and full scan range was set to $350-1600 \mathrm{~m} / \mathrm{z}$. We used a resolution of 60,000 at $\mathrm{m} / \mathrm{z}$ 200 for survey scans. Precursor ions were fragmented by high-energy collisional dissociation (NCE 27\%), and fragment ions were detected in the Orbitrap $(R=15,000$ at $m / z 200$ ). Dynamic exclusion duration was set to $30 \mathrm{~s}$.

\section{Peptide Identification}

The MS/MS data were converted into a Mascot Generic Format (MGF) file using Proteome Discoverer 1.4 (Thermo Fisher Scientific) for the Q Exactive HF and using MS Data Converter v1.3 (AB Sciex, Foster City, CA, USA) for the TripleTOF 5600 . The MGF data were searched by Mascot (version 2.3.02; MatrixScience, Boston, MA, USA) against our previous transcriptome database ${ }^{4}$. Enzyme was set to none, with Carbamidomethyl $(\mathrm{C})$ as a fixed modification, and Oxidation (M), Gln->pyro-Glu (N-term Q), Deamidated (NQ), 4-hydroxy-proline (P), 5-hydroxy-lysine (K), bromotryptophan (W), gamma-carboxy-glutamic acid (E), pyroglutamic acid (E), sulfotyrosine (Y), and gammahydroxy-D-valine (V) as variable modifications ${ }^{34}$. 
Decoy database was selected. Peptide mass tolerance was set to $10 \mathrm{ppm}$ and MS/MS tolerance was set to $0.02 \mathrm{Da}$ for the $\mathrm{Q}$ Exactive HF; peptide mass tolerance was set to $0.05 \mathrm{Da}$ and MS/MS tolerance was set to $0.1 \mathrm{Da}$ for the TripleTOF 5600.

Finally, the peptides were filtered at e-value $<0.05$ for the false discovery rate (FDR) using the Mascot searching engine; that is to say, the ion scores were higher than corresponding identity scores ${ }^{57,58}$. The manual method and the PepEx (https://github.com/ eparker05/PeptideExtractor) were both used to map the distribution of peptides onto their full-length conopeptide protein sequences. Protein modification sites and types were summarized by a perl script, and all possible combinations of the modified peptides are proposed on the basis of these determined modification parameters. PSM images were visualized by $\mathrm{PDV}^{59}$.

\section{Bioactivity prediction for determined conopeptides}

Based on the reported MVIIA, AuIB and ImI sequen$\mathrm{ces}^{23,24,60}$, homologous alignment was performed to screen peptides with potentially related activities from the determined 133 genes and 215 transcripts $^{4}$. These sequences were aligned for comparison by using MEGA6 and GeneDoc 2.7 (https://github.com/karlnicholas/ GeneDoc). To represent identical or homologous residues in each sequence, amino acids were marked with different colors.

The 3D structures of MVIIA, AuIB and ImI were downloaded from the public PDB database (http://www. wwpdb.org/). Models of the conopeptides were built using the MVIIA (PDB:1OMG), AuIB (PDB:1MXN) and ImI (PDB:2BC7) as templates in MODELLER (version $9 \mathrm{v} 12)^{61,62}$ as described previously ${ }^{8}$. All modeling images were generated with PyMol (http://www.pymol.org).

\section{Acknowledgements}

This work is supported by Shenzhen Special Project for High-Level Talents (No. SZYSGZZ-2018001), National Natural Science Foundation of China (No. 81560611), Key Research and Development Program of Hainan Province (No. ZDYF2018138), Shenzhen Grant Plan for Demonstration City Project for Marine Economic Development in Shenzhen (No. 86), and Shenzhen Dapeng Special Program for Industrial Development (No. KJYF202001-17).

\footnotetext{
Author details

'Shenzhen Key Lab of Marine Genomics, Guangdong Provincial Key Lab of Molecular Breeding in Marine Economic Animals, BGl Academy of Marine Sciences, BGl Marine, BGl, Shenzhen, Guangdong 518083, China. ${ }^{2}$ Key Laboratory of Tropical Translational Medicine of Ministry of Education, Hainan Provincial Key Laboratory of Research and Development of Herbs, School of Pharmacy, Hainan Medical University, Haikou, Hainan 570102, China. ${ }^{3}$ BGl Education Center, University of Chinese Academy of Sciences, Shenzhen, Guangdong 518083, China. ${ }^{4}$ Center of Reproduction, Development and Aging, Faculty of Health Sciences, University of Macau, Macau 999078, China. ${ }^{5}$ BGlShenzhen, BGl, Shenzhen, Guangdong 518083, China. ${ }^{6}$ China National GeneBank, BGl, Shenzhen, Guangdong 518120, China. ${ }^{7}$ Micro Pharmtech Ltd., Wuhan, Hubei 430000, China. ${ }^{8}$ Research Institute of Pharmaceutical Chemistry,

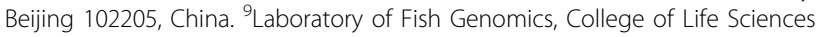
and Oceanography, Shenzhen University, Shenzhen, Guangdong 518061, China. ${ }^{10}$ Present address: College of Animal Science and Technology, Zhongkai
}

University of Agriculture and Engineering, Guangzhou, Guangdong 510225, China. ${ }^{11}$ Present address: College of Life Sciences, Neijiang Normal University, Neijiang, Sichuan 641100, China

\section{Author contributions}

Q.S. conceived the project. C.P., Y.H., C.B., J.Li., B.G., K.Z., and J.Liu. performed data analysis. X.Y., J.C., C.F., H.J., and J.S.C. prepared the samples. Z.L., Y.H., Z.X., Y.L., Y.Y., Z.R., Y.L., X.L., R.G., J.X., J.Y., and G.Y. participated in data analysis and figure preparation. C.P., J.Li, Y.H., J.Liu, B.G., and Q.S. wrote the manuscript. Q.S., C.P., and C.B. revised the manuscript. All authors approved submission of the final manuscript.

\section{Data availability}

Supporting datasets are included within this article and its Supplementary Information. The genome reads generated in this study have been deposited in China National GeneBank Nucleotide Sequence Archive (NSA) with accession IDs from CNX0040469 to CNX0040494 and from CNS0048937 to CNS0048939 under the project CNP0000362. The Whole Genome Shotgun project has been deposited at NCBI under the accession number JADBJO000000000 (Biosample: SAMN16261191; Bioproject: PRJNA665547). The version described in this paper is JADBJO010000000. The transcriptome reads have been previously deposited in the NCBI SRA database with accession numbers SRS1009725 (for the Big dataset), SRS1009729 (for the Middle dataset), SRS1009726 (for the Small dataset), SRS1009727 (for the Normalized dataset), and SRS1009728 (for the Bulb dataset). The clean reads for 11,026 clones were submitted to the NCBI as EST data (ID: PRJNA290540). All the mass spectrometry proteomics data have been submitted to the ProteomeXchange Consortium (http://www.proteomexchange.org/) via the PRIDE partner repository with the dataset ID PXD014892.

\section{Conflict of interest}

The authors declare that they have no conflict of interests.

\section{Publisher's note}

Springer Nature remains neutral with regard to jurisdictional claims in published maps and institutional affiliations.

Supplementary information The online version contains supplementary material available at https://doi.org/10.1038/s41421-021-00244-7.

Received: 9 July 2020 Accepted: 29 December 2020

Published online: 23 February 2021

\section{References}

1. Gao, B. et al. Cone snails: a big store of conotoxins for novel drug discovery. Toxins 9, 397 (2017).

2. Gao, B. et al. Mitochondrial genome sequencing of a vermivorous cone snail Conus quercinus supports the correlative analysis between phylogenetic relationships and dietary types of Conus species. PLoS ONe 13, e0193053 (2018).

3. Gao, B. et al. High throughput identification of novel conotoxins from vermivorous Oak cone snail (Conus quercinus) by transcriptome sequencing. Int. J. Mol. Sci. 19, 3901 (2018).

4. Peng, C. et al. High-throughput identification of novel conotoxins from the Chinese tubular cone snail (Conus betulinus) by multi-transcriptome sequencing. Gigascience 5, 17 (2016).

5. Terlau, H. \& Olivera, B. M. Conus venoms: a rich source of novel ion-channeltargetd peptides. Physiol. Rev. 84, 41-68 (2004).

6. McGivern, J. G. Ziconotide: a review of its pharmacology and use in the treatment of pain. Neuropsych. Dis. Treat. 3, 69-85 (2007).

7. Woodwasr, S. R. et al. Constant and hypervariable regions in conotoxin propeptides. EMBO J. 9, 1015-1020 (1990).

8. Gao, B. et al. Screening and validation of high-efficient insecticidal conotoxins from a transcriptome-based dataset in Chinese tubular cone snail. Toxins $\mathbf{9}$, 214 (2017)

9. Dutertre, S. et al. Deep venomics reveals the mechanism for expanded peptide diversity in cone snail venom. Mol. Cell. Proteom. 12, 312-329 (2013). 
10. Kaas, Q. et al. Conopeptide characterization and classifications: an analysis using ConoServer. Toxicon 55, 1491-1509 (2010).

11. Davis, J. et al. Remarkable inter- and intra-species complexity of conotoxins revealed by LC/MS. Peptides 30, 1222-1227 (2009).

12. Biass, D. et al. Comparative proteomic study of the venom of the piscivorous cone snail Conus consors. J. Proteom. 72, 210-218 (2009).

13. $\mathrm{Hu}, \mathrm{H}$. et al. Characterization of the Conus bullatus genome and its venomduct transcriptome. BMC Genomics 12, 60 (2011).

14. Phuong, M. A. \& Mahardika, G. N. Targeted sequencing of venom genes from cone snail genomes improves understanding of conotoxin molecular evolution. Mol. Biol. Evol. 35, 1210-1224 (2018).

15. Song, L. et al. Draft genome of the Chinese mitten crab, Eriocheir sinensis. GigScience 5, 5 (2016).

16. Li, C. et al. Draft genome of the Peruvian scallop Argopecten purpuratus. Gigascience 7, giy031 (2018).

17. Waterhouse, R. M. et al. BUSCO applications from quality assessments to gene prediction and phylogenomics. Mol. Biol. Evol. 35, 543-548 (2017).

18. Rao, S. S. et al. A 3D map of the human genome at kilobase resolution reveals principles of chromatin looping. Cell 159, 1665-1680 (2014).

19. Bolger, A. et al. Trimmomatic: a flexible trimmer for Illumina sequence data. Bioinformatics 30, 2114-2120 (2014).

20. Durand, N. C. et al. Juicer provides a one-click system for analyzing loopresolution Hi-C experiments. Cell Syst. 3, 95-98 (2016).

21. Dalet, J. T. et al. Karyological analysis and FISH physical mapping of $18 \mathrm{~S}$ rDNA genes, (GATA)n centromeric and (TTAGGG)n telomeric sequences in Conus magus Linnaeus, 1758. J. Mollusca. Stud. 81, 274-289 (2015).

22. Luo, S. et al. Alpha-conotoxin AulB selectively blocks alpha3 beta4 nicotinic acetylcholine receptors and nicotine-evoked norepinephrine release. J. Neurosci. 18, 8571-8579 (1998).

23. $\mathrm{Yu}, \mathrm{R}$. et al. Delineation of the unbinding pathway of a-conotoxin $\mathrm{Iml}$ from the a7 nicotinic acetylcholine receptor. J. Phys. Chem. B 116, 6097-6105 (2012).

24. $\mathrm{Wu}, \mathrm{X}$. et al. Stoichiometry dependent inhibition of rat a3ß4 nicotinic acetylcholine receptor by the ribbon isomer of a-conotoxin AulB. Biochem. Pharmacol. 155, 288-297 (2018).

25. Barghi, N. et al. Structural features of conopeptide genes inferred from partial sequences of the Conus tribblei genome. Mol. Genet. Genomics 291, 411-422 (2015)

26. Dutertre, $\mathrm{S}$. et al. Dramatic intraspecimen variations within the injected venom of Conus consor: an unsuspected contribution to venom diversity. Toxicon $\mathbf{5 5}$ 1453-1462 (2010)

27. Terrat, Y. et al. High-resolution picture of a venom gland transcriptome: case study with the marine snail Conus consors. Toxicon 59, 34-46 (2012).

28. Himaya, S. W. et al. Comparative venomics reveals the complex prey capture strategy of the piscivorous cone snail Conus catus. J. Proteome Res. 14 4372-4381 (2015).

29. Prashanth, J. R. et al. The role of defensive ecological interactions in the evolution of conotoxins. Mol. Ecol. 25, 598-615 (2016).

30. Dutertre, S. et al. Evolution of separate predation- and defence-evoked venoms in carnivorous cone snails. Nat. Commun. 5, 3521 (2014).

31. Wilkins, M. R. et al. Protein identification and analysis tools in the ExPASy server. Methods Mol. Biol. 112, 531-552 (1999).

32. Li, R. et al. SOAP2: an improved ultrafast tool for short read alignment. Bioinfomatics 25, 1966-1967 (2009).

33. Ye, C. et al. Exploiting sparseness in de novo genome assembly. BMC Bioinforma. 13, S1 (2012)

34. Ye, C. et al. DBG2OLC: efficient assembly of large genomes using long erroneous reads of the third generation sequencing technologies. Sci. Rep. $\mathbf{6}$, 31900 (2016)

35. Li, H. Minimap2: pairwise alignment for nucleotide sequences. Bioinformatics 34, 3094-3100 (2018).

36. Vaser, R. et al. Fast and accurate de novo genome assembly from long uncorrected reads. Genome Res. 27, 737-746 (2017).
37. Boetzer, M. et al. Scaffolding pre-assembled contigs using SSPACE. Bioinformatics 27, 578-579 (2011)

38. Boetzer, M. \& Pirovano, W. SSPACE-LongRead: scaffolding bacterial draft genomes using long read sequence information. BMC Bioinforma. 15, 211 (2014).

39. Tarailo-Graovac, M. \& Chen, N. Using RepeatMasker to identify repetitive elements in genomic sequences. Curr. Protoc. Bioinforma. 25, 4.10.1-4.10.14 (2009).

40. Jurka, J. et al. Repbase Update, a database of eukaryotic repetitive elements. Cytogenet. Genome Res. 110, 462-467 (2005).

41. Xu, Z. \& Wang, H. LTR_FINDER: an efficient tool for the prediction of full-length LTR retrotransposons. Nucleic Acids Res. 35, W265-W268 (2007).

42. Benson, $\mathrm{G}$. Tandem repeats finder: a program to analyze DNA sequences. Nucleic Acids Res. 27, 573-580 (1999).

43. Holt, C. \& Yandell, M. MAKER2: an annotation pipeline and genome-database management tool for second-generation genome projects. BMC Bioinforma. 12, 491 (2011).

44. Mount, D. W. Using the Basic Local Alignment Search Tool (BLAST). CSH Protoc. 2007, pdb top17 (2007).

45. Birney, E. et al. GeneWise and Genomewise. Genome Res. 14, 988-995 (2004).

46. Barghi, N. et al. High conopeptide diversity in Conus tribblei revealed through analysis of venom duct transcriptome using two high-throughput sequencing platforms. Mar. Biotechnol. 17, 81-98 (2015).

47. Corpet, F. Multiple sequence alignment with hierarchical clustering. Nucleic Acids Res. 16, 10881-10890 (1988).

48. Tamura, K. et al. MEGA6: Molecular Evolutionary Genetics Analysis version 6.0. Mol. Biol. Evol. 30, 2725-2729 (2013).

49. Li, R. et al. The sequence and de novo assembly of the giant panda genome Nature 463, 311-317 (2010).

50. Dudchenko, O. et al. De novo assembly of the Aedes aegypti genome using Hi-C yields chromosome-length scaffolds. Science 356, 92-93 (2017).

51. Durand, N. C. et al. Juicebox provides a visualization system for $\mathrm{Hi}-\mathrm{C}$ contact maps with unlimited zoom. Cell Syst. 3, 99-101 (2016).

52. Aguilar, M. B. et al. A novel conotoxin from Conus delessertii with posttranslationally modified lysine residues. Biochemistry 44, 11130-11136 (2005).

53. Van Der Haegen, A. et al. Pc16a, the first characterized peptide from Conus pictus venom, shows a novel disulfide connectivity. Peptides 34, 106-113 (2012).

54. Peigneur, S. et al. Unraveling the peptidome of the South African cone snails Conus pictus and Conus natalis. Peptides 41, 8-16 (2013).

55. Luan, N. et al. A combinational strategy upon RNA sequencing and peptidomics unravels a set of novel toxin peptides in scorpion Mesobuthus martensii. Toxins 8, 286 (2016).

56. Ruperez, P. et al. Quantitative phosphoproteomic analysis reveals a role for serine and threonine kinases in the cytoskeletal reorganization in early $\mathrm{T}$ cell receptor activation in human primary T cells. Mol. Cell. Proteom. 11, 171-186 (2012).

57. Elisa, J. E. \& Gygi, S. P. Target-decoy search strategy for increased confidence in large-scale protein identifications by mass spectrometry. Nat. Methods 4 207-214 (2007)

58. Kochanowski, M. et al. Proteomic and bioinformatic investigations of heattreated anisakis simplex third-stage larvae. Biomolecules 10, 1066 (2020).

59. Li, K. et al. PDV: an integrative proteomics data viewer. Bioinformatics $\mathbf{3 5}$, 1249-1251 (2019)

60. Rigo, F. K. et al. Effect of $\omega$-conotoxin MVIIA and Pha1 $\beta$ on paclitaxel-induced acute and chronic pain. Pharmacol. Biochem. Behav. 114, 16-22 (2013).

61. Sali, A. \& Blundell, T. L. Comparative protein modelling by satisfaction of spatial restraints. J. Mol. Biol. 234, 779-815 (1993).

62. Webb, B. \& Sali, A. Comparative protein structure modeling using MODELLER Curr. Protoc. Bioinforma. 54, 5.6.1-5.6.37 (2016). 